\title{
Correlation of oscillatory behaviour in Matlab using wavelets
}

\author{
T.D. Pering ${ }^{\mathrm{a}, *}$, G. Tamburello ${ }^{\mathrm{b}}$, A.J.S. McGonigle ${ }^{\mathrm{a}, \mathrm{c}}$, E. Hanna ${ }^{\mathrm{a}}$, A. Aiuppa ${ }^{\mathrm{b}, \mathrm{c}}$ \\ a University of Sheffield, Department of Geography, Winter Street, S10 2TN, United Kingdom \\ b DiSTeM, Università di Palermo, via Archirafi, 22, 90123 Palermo, Italy \\ ' Istituto Nazionale di Geofisica e Vulcanologia, Sezione di Palermo, Via Ugo La Malfa, 153, 90146 Palermo, Italy
}

\section{A R T I C L E I N F O}

\section{Article history:}

Received 17 December 2013

Received in revised form

7 June 2014

Accepted 10 June 2014

Available online 18 June 2014

Keywords:

Continuous wavelet transform

Wavelets

Spearman's rank correlation

Periodicity

Oscillation

De-noising

\begin{abstract}
A B S T R A C T
Here we present a novel computational signal processing approach for comparing two signals of equal length and sampling rate, suitable for application across widely varying areas within the geosciences. By performing a continuous wavelet transform (CWT) followed by Spearman's rank correlation coefficient analysis, a graphical depiction of links between periodicities present in the two signals is generated via two or three dimensional images. In comparison with alternate approaches, e.g., wavelet coherence, this technique is simpler to implement and provides far clearer visual identification of the inter-series relationships. In particular, we report on a Matlab ${ }^{\circledR} \operatorname{code}$ which executes this technique, and examples are given which demonstrate the programme application with artificially generated signals of known periodicity characteristics as well as with acquired geochemical and meteorological datasets.
\end{abstract}

(c) 2014 Elsevier Ltd. All rights reserved.

\section{Introduction}

Given the significant increase in computational power over the last decades, signal processing techniques such as wavelet analysis have become common place in their application within the geosciences. In particular, wavelets are applied, via a process of convolution, to reveal information on periodicities present in data series, and their stability as a function of time, in contrast to Fourier transforms, which only probe frequency characteristics (Welch, 1967; Harris, 1978). The exception here is with the Short Fourier Transform (e.g., spectrogram), which is applied to reveal spectral frequency variations with time (Oppenheim et al., 1999). Whereas, a continuous wavelet transform (CWT) operates over a continuous range of scales, providing potentially more detailed information than the discretely sampled discrete wavelet or Short Fourier Transform (Torrence and Compo, 1998; Oppenheim et al., 1999). Hence, wavelets are more suited to investigation of transient or unstable periodic phenomena.

Oscillatory behaviour is widely manifest in datasets acquired from across the geo and environmental sciences, for example concerning the 11-year sunspot cycle (e.g. Hoyt and Schatten, 1997; Frohlich and Lean, 2004), the El Niño Southern Oscillation (Torrence and Compo, 1998) and the North Atlantic Oscillation (NAO) (Hurrell, 1995). These phenomena can change significantly

\footnotetext{
* Corresponding author: Tel.: +44 7838219369.

E-mail address: ggp12tdp@sheffield.ac.uk (T.D. Pering).
}

in strength and period as a function of time and are an integral part of climate variability (e.g. Hurrell et al., 2003; Lockwood, 2012; Philander, 1990). Oscillations are also present over much shorter timescales of seconds to hours, for example within geochemical datasets concerning volcanic degassing (Tamburello et al., 2012). The links between fluctuations present in environmental data series can wax and wane dramatically, providing a motivation for the application of wavelet analysis. Here we present a straightforward and new approach to investigating the correlation between oscillations present in two or more environmental datasets; this technique is based on CWT analysis using Matlab ${ }^{\mathbb{R}}$ and the Matlab Wavelet Toolbox ${ }^{\circledR}$ followed by Spearman's rank correlation coefficient analysis.

\section{Technique overview}

The Matlab ${ }^{\circledR}$ function (available in the auxiliary materials) was written in Matlab ${ }^{\circledR}$ 2010b and has been tested on the 2008a, 2011b and 2013a versions, with correct operation demonstrated in each case. The programme uses the CWT function (part of the Matlab Wavelet Toolbox ${ }^{\circledR}$ ) for two separate signals. These signals should be normalised prior to processing by this code, however, the code performance is independent of the applied normalisation technique. This is followed by linear correlation (using Spearman's rank correlation coefficient, which accounts for non-linearity and variable amplitude of the wavelet coefficients), to generate a visual representation of the links between the coefficients generated by 
a

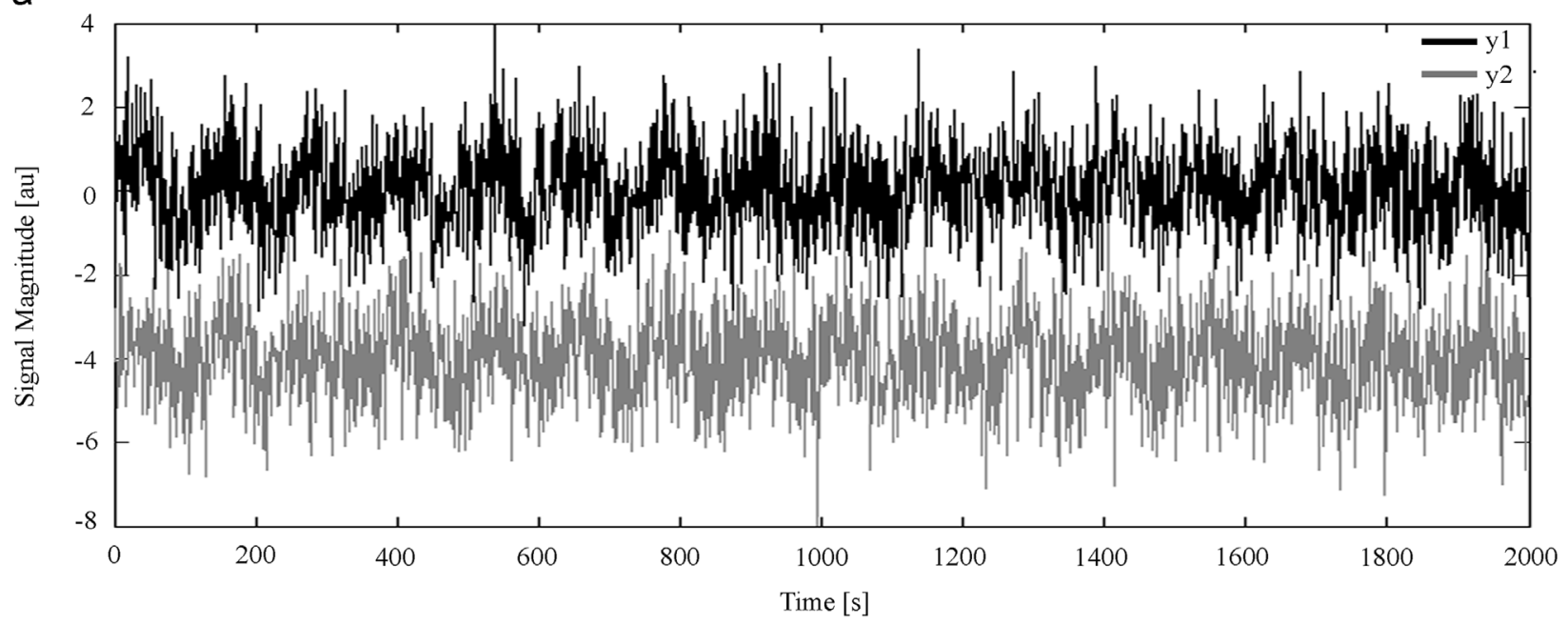

b

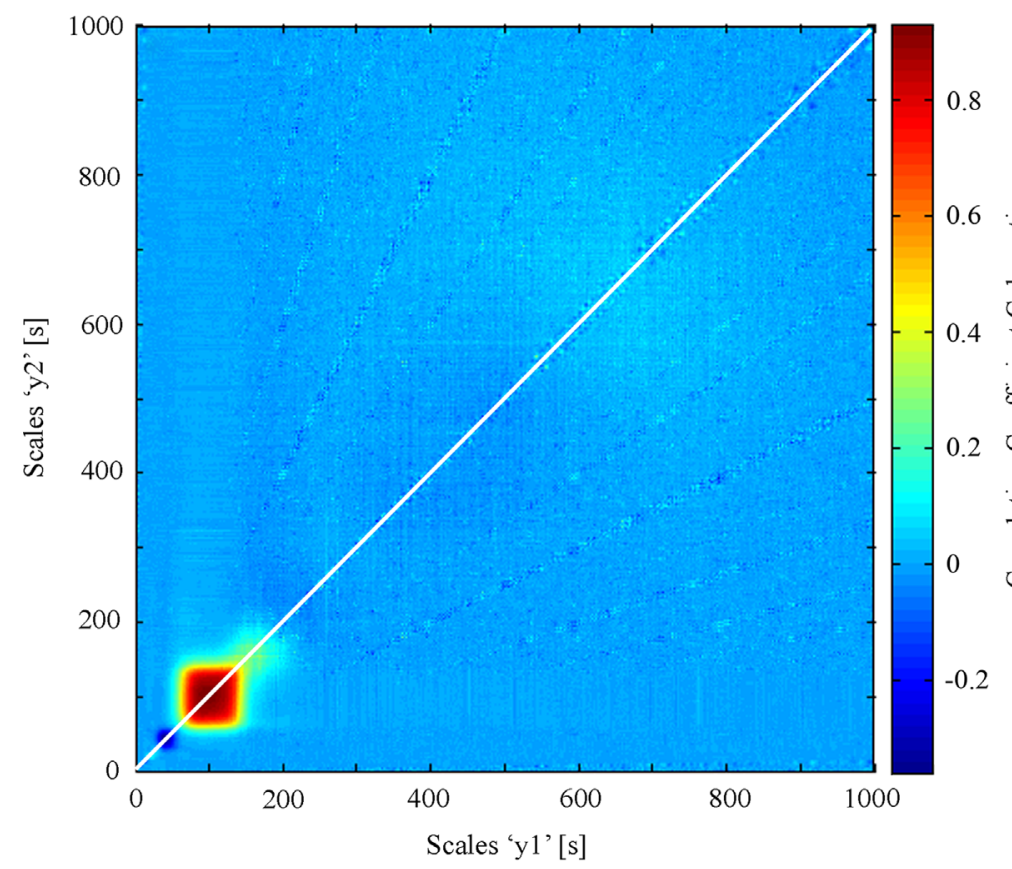

C

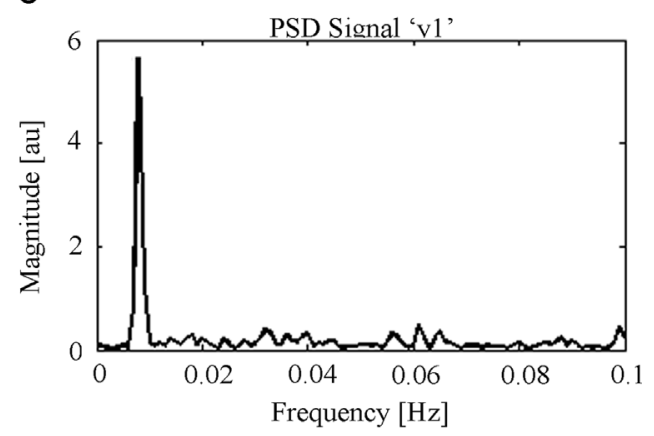

d

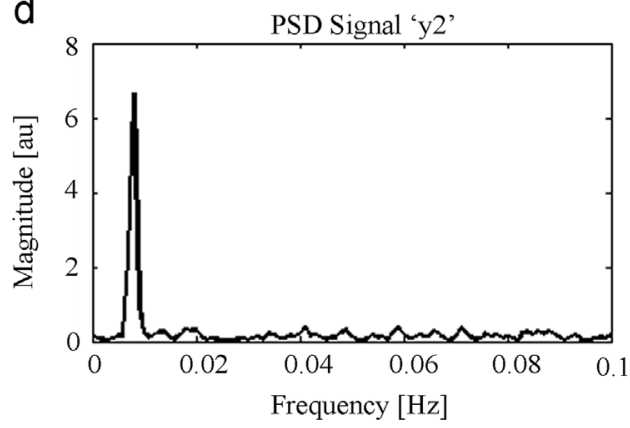

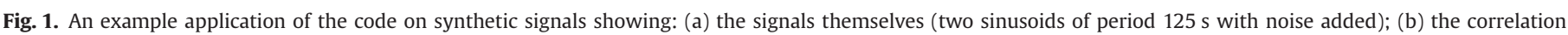

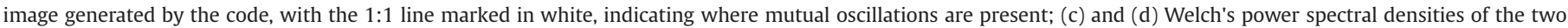
series, which show the dominant oscillation at $125 \mathrm{~s}$ in each case.

the wavelet transforms (e.g. Figs. 1b, 3d, 4, 5a, b). For the examples illustrated in this paper the Morlet wavelet was applied as the mother wavelet (Morlet et al., 1982; Grinstead et al., 2004)

$\Psi_{0}(\eta)=\pi^{-1 / 4} e^{i \omega_{0} \eta} e^{-\eta^{2} / 2}$.

where $\Psi_{0}(\eta)$ is the wavelet function, $\eta$ is a non-dimensional parameter representing a time component and $\omega_{0}$ refers to the wavelets' non-dimensional frequency. This particular class of wavelet is implemented here, given its similarity to naturally occurring oscillations manifest in data series spanning the geosciences (e.g. Torrence and Compo, 1998). This said, the code could also use non-complex alternates, e.g., Gaussian wavelets from the Matlab Wavelet Toolbox ${ }^{\circledR}$ if these are judged more suitable for the application in question. Indeed, the Matlab Wavelet Toolbox ${ }^{\circledR}$ provides a comprehensive overview and visualisation of available mother wavelets. In general, wavelet analysis works best with selection of a mother wavelet which closely resembles the target oscillation. The CWT itself is defined as (e.g. Grinstead et al., 2004)

$$
W_{n}(s)=\sqrt{\frac{\delta t}{s}} \sum_{n^{\prime}=1}^{N} x_{n^{\prime}} \Psi^{*}\left[\left(n^{\prime}-n\right) \frac{\delta t}{s}\right],
$$

where $\delta t$ is a uniform time-step, $x_{n}$ is the subject signal, $W_{n}(s)$ represents the changing wavelet scale on the left-hand-side and similarly as $s$ on the right-hand-side, $*$ is the complex conjugate, $N$ is the maximum scale, and $n$ is the points of the time series, (Morlet et al., 1982; Colestock, 1993; Grinstead et al., 2004). The result is the conjugation of the scaled selected wavelet with the subject signal and outputs, which demonstrates the stability and power of any periodic features which match the scaled wavelet. We refer to the extensive literature for more in-depth descriptions of the CWT (e.g. Morlet et al., 1982; Daubechies, 1990; Colestock, 1993; Huang et al., 1998; Torrence and Compo, 1998). 
The next step is to correlate the output of the CWT at each scale $\left(W_{n i}\right)$ using Spearman's Rank $\left(r_{s}\right)$ correlation coefficient (Spearman, 1904; Zar, 1972)

$r_{s}\left(W_{n i}\right)=1-\frac{6 \sum d_{i}^{2}\left(W_{n i}\right)}{n\left(n^{2}-1\right)}$,

where $d_{i}^{2}$ is the ranked difference between the outputs of each CWT. The code, therefore, determines the degree of match between oscillations present in the two different signals over a broad scale range. This is particularly useful where signals are highly variable or 'noisy' and where links are difficult to discern from comparison of the individual standard wavelet transforms. Likewise, this provides clearer scope for visual identification of

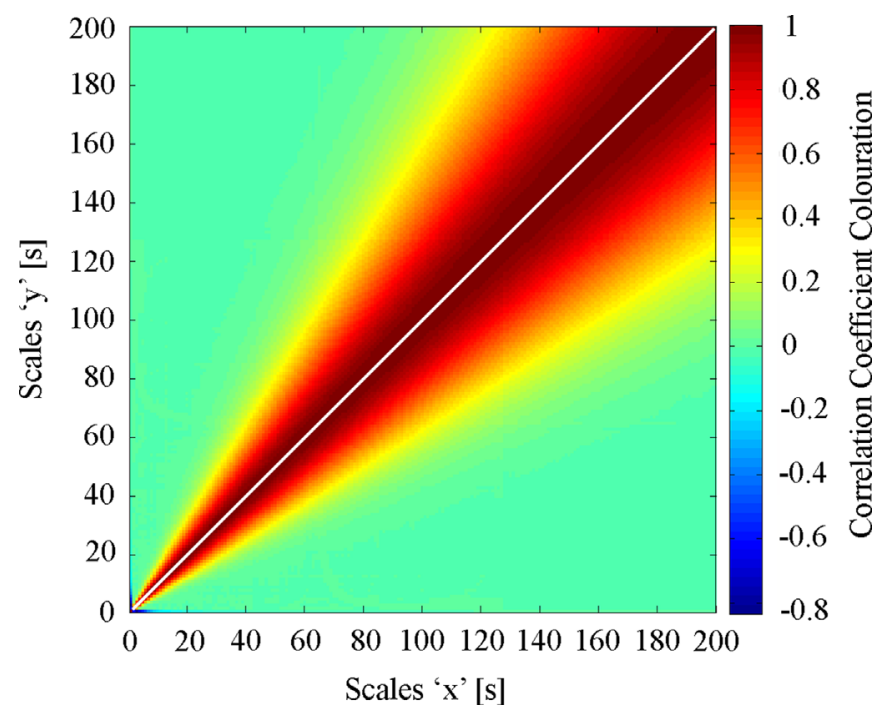

Fig. 2. A sample correlation image for perfect correlation over all scales. links between the series than alternates such as wavelet coherence (e.g., Grinstead et al., 2004; Cannata et al., 2013) by virtue of generating a single plot whose axes are the scales of the compared datasets, rather than two discrete plots of scales vs. time. This approach also requires less computational power, in addition to the primary benefits of the technique, namely: simplicity of operation and ease in interpretation. This is a code and display approach, which to the authors' knowledge, has not previously been applied or documented in the literature, with the exception of a brief overview given in Pering et al. (2014).

\section{The Matlab ${ }^{\circledR}$ function}

In summary, the Matlab function 'corrplot.m' is displayed below, including only those elements related to the production and extraction of data. The full code is available online in the supplementary materials. The code requires a number of inputs: signals $x$ and $y$ (e.g., the data series which are to be compared, which must be of identical sampling frequency and length); wavelet type (e.g., the class of mother wavelet, for example 'morl' for Morlet); scales (e.g., the maximum scale for the CWT - the default setting is to run the CWT in steps of 1 , from 1 up to this value); and finally, the sampling rate of the dataset in Hertz (Hz). The dominant oscillation(s) in each of the input series are also determined as part of the code, using Welch's power spectral density (PSD) method (Welch, 1967), as an additional means of identifying similarities in the series. Furthermore, an automatic code-interruption error message is incorporated to avoid analysis above the Nyquist criterion (Nyquist, 2002).

$$
\begin{gathered}
\text { function }[\mathrm{a}, \mathrm{b}]=\operatorname{corrplot}(\mathrm{x}, \mathrm{y}, \text { wavelet,scales,fs }) \\
\text { if scales }>((\text { length }(x) / 2)) \\
\text { error('Scales above Nyquist limit') } \\
\text { end }
\end{gathered}
$$

\% Wavelet Transform
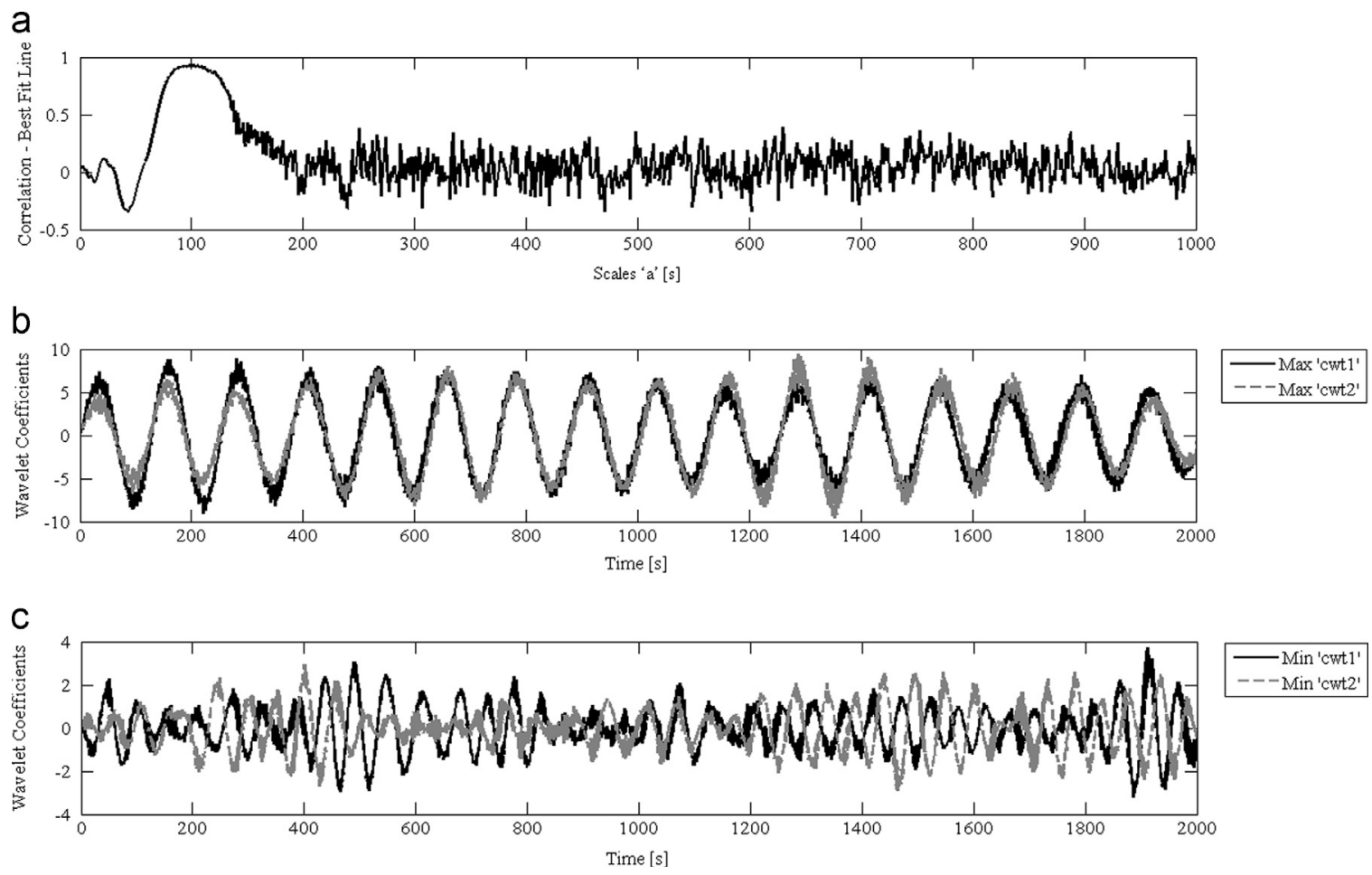

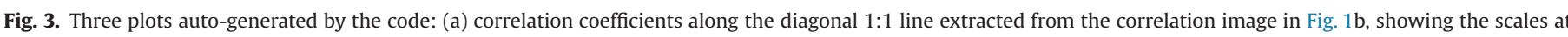

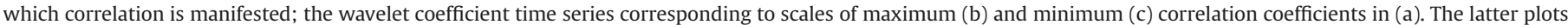
allow the user to investigate temporal lags between the series, in this case confirming that the two series have a mutual in phase oscillation at $125 \mathrm{~s}$. 

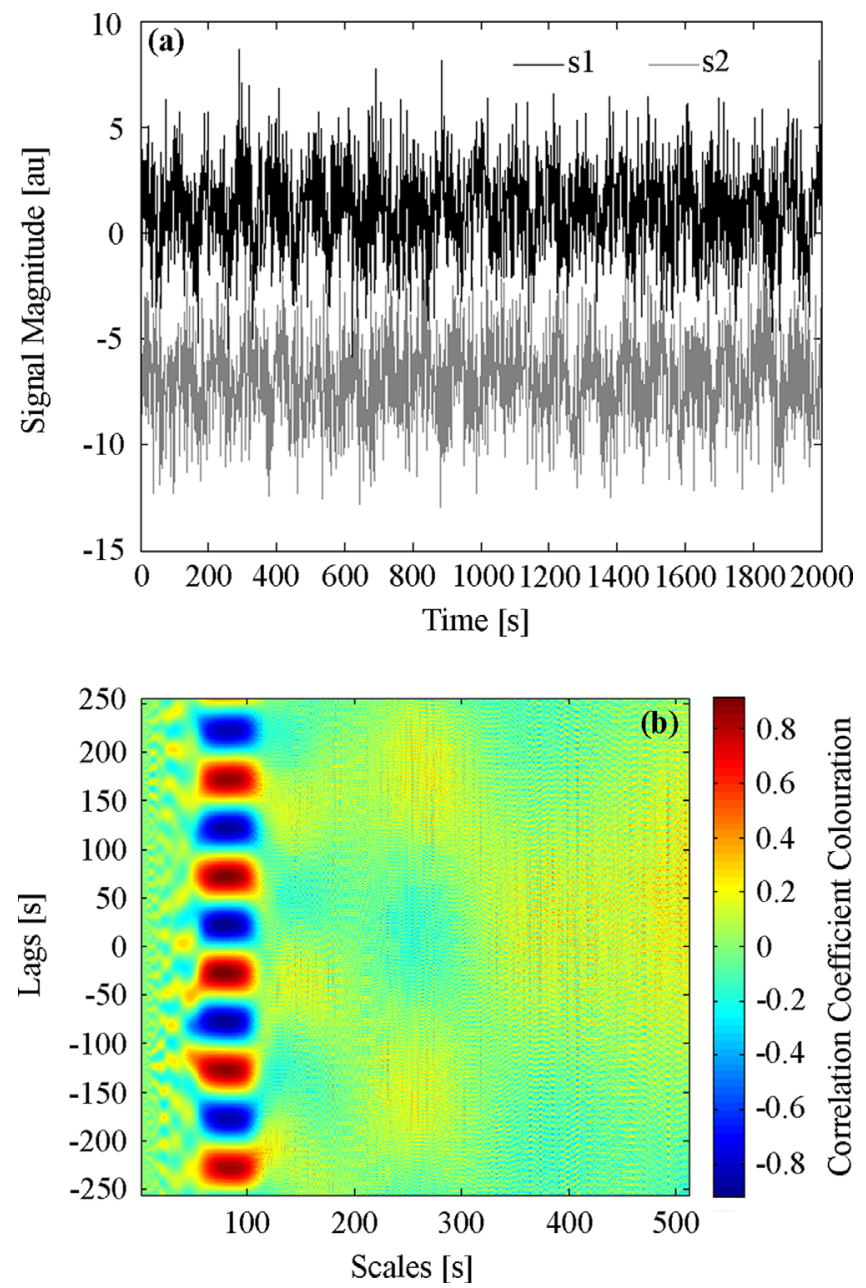

Fig. 4. An example application of the code on: (a) cosinusoidal (s1) and sinusoidal (s2) signals, with matching period of $90 \mathrm{~s}$ and added random noise. In (b) the last auto-generated plot by the code shows the correlation coefficients at the given lag value and wavelet coefficient scale. The latter plot is of particular use for determining lags, in addition to those in Fig. 3, and also when signals are not in perfect phase or antiphase.

cwt1 $=\operatorname{cwt}(\mathrm{x} / \max (\mathrm{x}), 1$ :scales, wavelet $)$;

cwt2 $=\operatorname{cwt}(\mathrm{y} / \max (\mathrm{y}), 1$ :scales,wavelet $) ;$

$\%$ Shift the data

cwt $1=$ ctranspose $($ cwt 1$)$; cwt $2=$ ctranspose $(\mathrm{cwt} 2)$;

$\%$ Correlate the data $\mathrm{a}=$ corr(cwt1,cwt2,'type','Spearman');

\% Extract the "best-fit" line $\mathrm{b}=\operatorname{diag}(\mathrm{a})$;

\% Extract max and min correlation location [max_corr,loc_max_corr] $=\max (\mathrm{b})$

[min_corr,loc_min_corr] $=\min (\mathrm{b})$

[M1,N1] =ind2sub(size(b),loc_max_corr);

[M2,N2] = ind2sub(size(b),loc_min_corr);

$\%$ Individual coefficients at max and min location

wave_coeff1_max=cwt1(:,M1); wave_coeff1_min=cwt1(:,M2);

wave_coeff2_max $=$ cwt2(:,M1); wave_coeff2_min $=$ cwt2(:,M2);

$\%$ Power spectral densities

[b1,freq1] = pwelch $(x / \max (\mathrm{x})$, scales,0,scales,fs $)$;

$[\mathrm{b} 2$, freq 1$]=\operatorname{pwelch}(\mathrm{y} / \max (\mathrm{y})$, scales, 0, scales,fs $)$;

$\%$ Xcorr lag plot

cwt1 = ctranspose $(\mathrm{cwt} 1)$;

cwt2 = ctranspose $(\mathrm{cwt} 2)$;

for $1 \mathrm{~s}=1$ :scales;

$$
\mathrm{s} 1=\operatorname{cwt} 1(\mathrm{ls},:) \text {; }
$$

$\mathrm{s} 2=\mathrm{cwt} 2(\mathrm{ls}, \mathrm{)})$;

maxlags $=$ scales $/ 2$;

lag_corr =xcorr(s1,s2,maxlags, 'coeff');

end

$\mathrm{c}(\mathrm{ls},:)=$ horzcat(lag_corr);

$\mathrm{c}=$ ctranspose $(\mathrm{c})$;

The code generates the following outputs: of which, the first, fourth and sixth can be exported to the Matlab ${ }^{\circledR}$ workspace

(i) a correlation image with colour scale;

(ii) power spectral densities of signals ' $x$ ' and ' $y$ ';

(iii) a $3 \mathrm{D}$ visualisation of the correlation image;

(iv) correlation coefficients along the $1: 1$ line in the correlation image;

(v) plots of the wavelet coefficients, which correspond to the points of maximum positive and negative correlations, along with 1:1 line; and

(vi) a plot with colour scale showing the correlation coefficients of the wavelet coefficients at each individual scale, over a defined range of lags.

\section{Example applications}

Firstly, we present an example application of the code on a pair of synthetic signals to illustrate this approach for establishing the presence of common periodicities. Fig. 1a shows these signals: two sinusoids of period $125 \mathrm{~s}$, with noise added, using a normally distributed random number generator. The generated 2D correlation image (Fig. 1b) shows a clear positive correlation between $\approx 75$ and $150 \mathrm{~s}$, with a peak value $>0.8$, and the dominant series frequencies are further manifest in the Welch's PSD curves in Fig. 1c and d showing a clear peak at $125 \mathrm{~s}(0.008 \mathrm{~Hz})$ in each case. The correlation plot also demonstrates that there are no other sources of significant correlation on any other timescales. For reference, a correlation image showing perfect correlations across all scales is presented in Fig 2. Probability values for observed correlations can be easily estimated using in-built Matlab ${ }^{\circledR}$ algorithms e.g., see Kendall (1970), Best and Roberts (1975), Ramsey (1989), and references therein for additional information.

The 1:1 line is included in Figs. $1 \mathrm{~b}$ and 2 to highlight the region in which one would expect relationships to occur e.g., where periods are common to both series. Fig. 3a shows the coefficient profile along this line, auto-generated by 'corrplot.m' from the correlation image (Fig. 1b): revealing the scales at which correlation is manifested in this case. It is then for the user to investigate the cause of such links, e.g., through analysis of whether the series are in or out of phase or shifted in phase relative to one another. To expedite this, the code also extracts the wavelet coefficient time series for the scales along the 1:1 line which present the strongest points of maximum and minimum correlation; these outputs are shown in Fig. 3b and c, respectively, for our sample synthetic data. In this case, the in-phase nature of the two $125 \mathrm{~s}$ period sinusoids is clearly manifested in Fig. 3a. For series which are out of phase, the lag could be determined by visual inspection of these two wavelet coefficient time series. As an additional aid, the code outputs the cross-correlation coefficient at each wavelet coefficient scale over the maximum possible range of lags. The code produces an image (e.g., Fig. 4) which clearly indicates the maximum or minimum lag between the series at each scale. This is of particular use when the signals are not perfectly in phase or antiphase. This capability of the code is illustrated on a cosinusoidal (s1) and sinusoidal (s2) signal (Fig. 4a), both generated with the same 

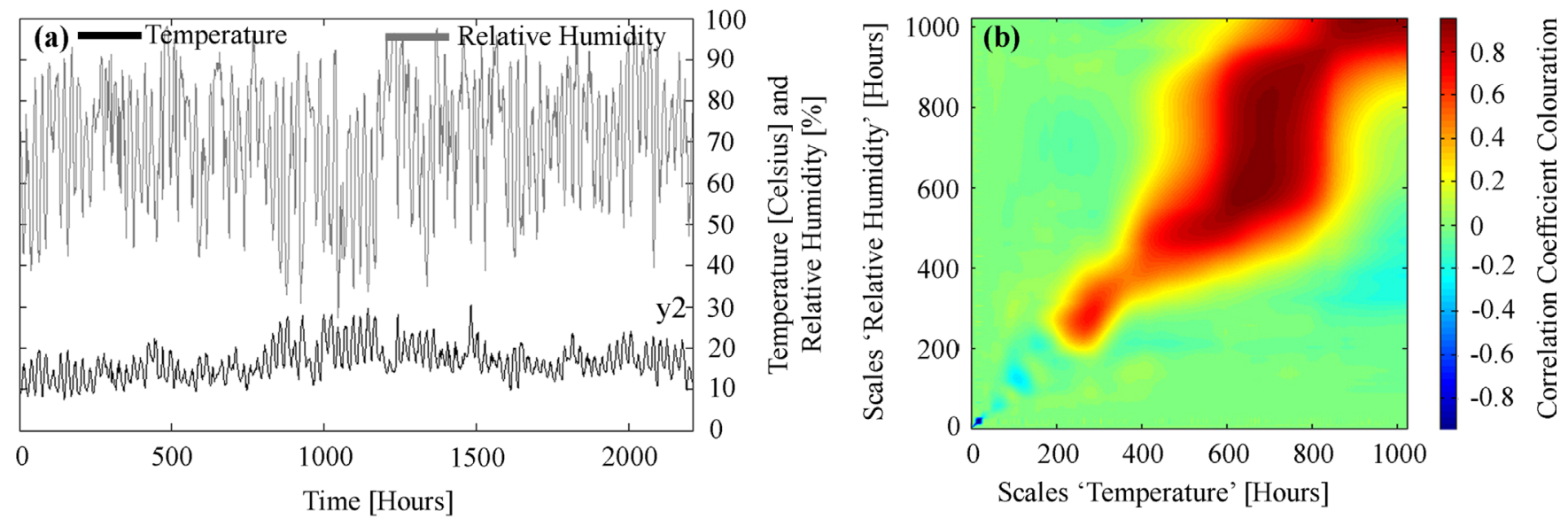

Normalised Coefficients for Wavelet Plots

0

\section{1}
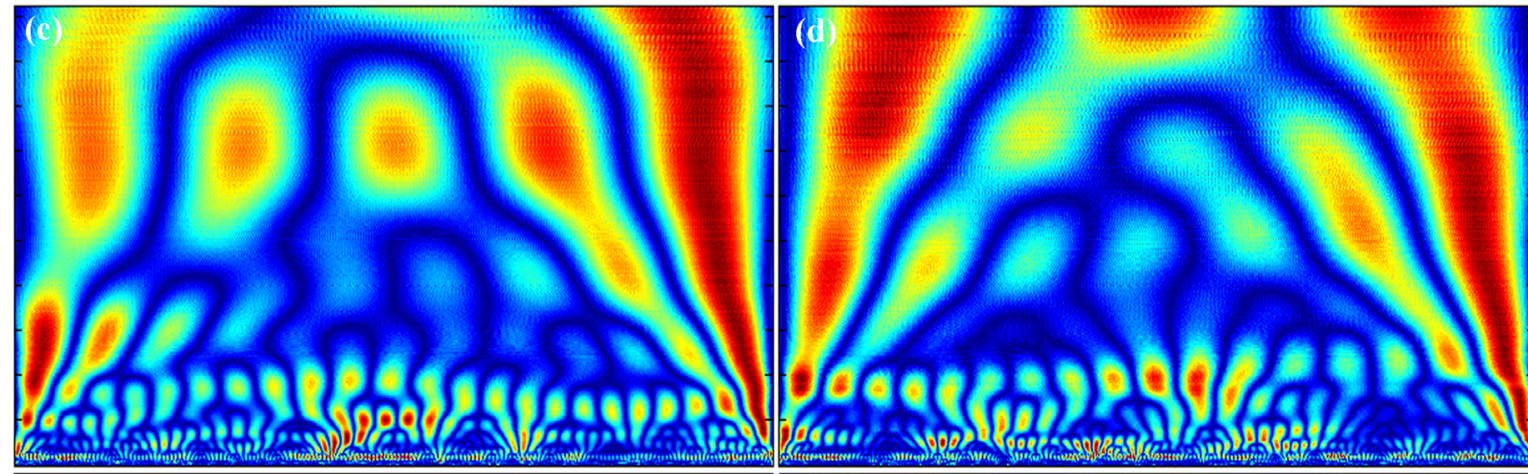

1000
900
800
700
600
500
400
300
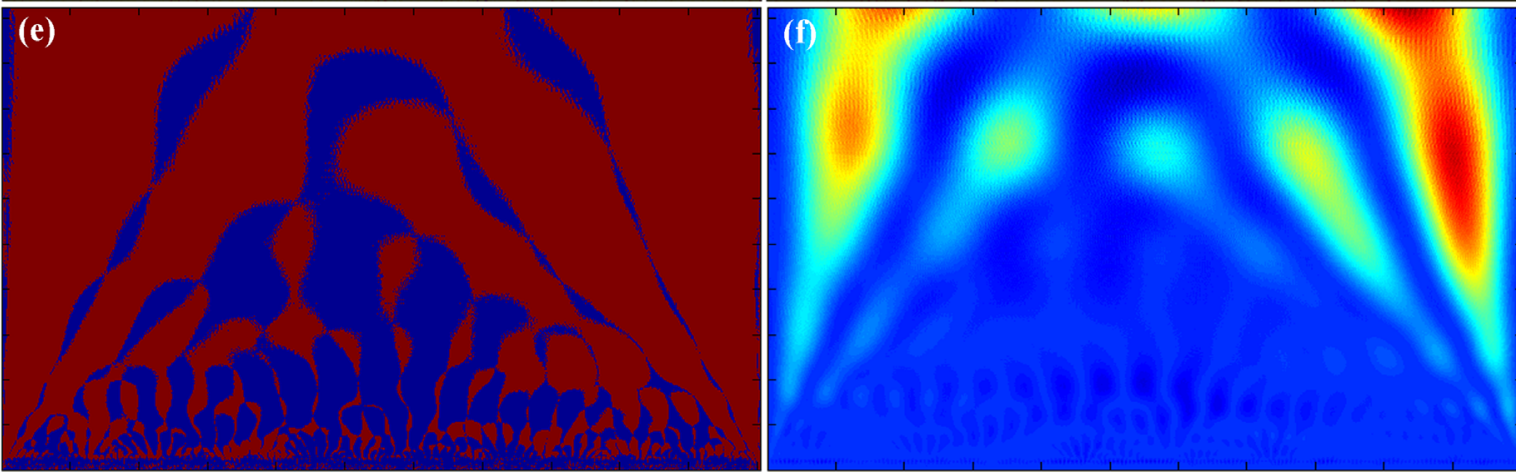

200

100

1000

900

800

700

600

500

400

300

200

100

$0 \quad 200 \quad 400 \quad 600 \quad 800 \quad 100012001400160018002000$

$0 \quad 200 \quad 400 \quad 600 \quad 8001000120014001600180020002200$

Time [Hours]

Time [Hours]

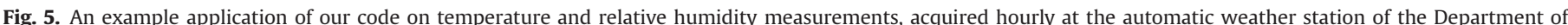

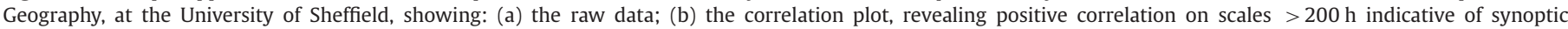

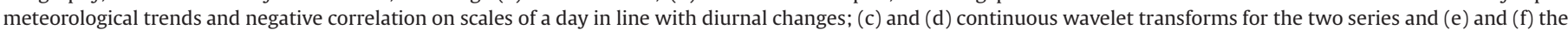

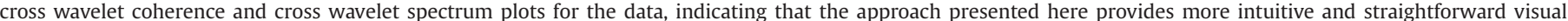
identification of the inter-series links, than available from these alternatives.

frequency (90 s) and amplitude, and with added random noise. The possible lags can be identified in Fig. $4 \mathrm{~b}$, the spacing between which clearly correspond to the known signals' frequency value. These particular functions are of particular use for investigating the links and lags between oscillations and periodicity in natural contexts, where raw signals can demonstrate considerable temporal variability.

We also applied the 'corrplot.m' code to measurements of temperatures and relative humidity collected hourly from the Department of Geography, University of Sheffield automatic weather station during June, July and August 2013. The raw data are presented in Fig. 5a and the resulting correlation image is shown in Fig. 5b, facilitating straightforward identification of the links present between the two data series. As expected, strong relationships are present at periods $>200$ h (e.g., $>8$ days), with peak correlation values at $\approx 600-800 \mathrm{~h}$ (e.g., $\approx 25-33$ days). This demonstrates that our technique clearly resolves the inter-series links related to synoptic meteorological changes occurring on timescales of weeks. Furthermore, a strong link, of $r_{s}=-0.94$ at $\approx 24 \mathrm{~h}$ is evident, capturing the relationships between changes in temperature and humidity over the diurnal cycle.

For comparison, the continuous wavelet transform plots of these two series are presented in Fig. 5c and d. The cross wavelet coherence and the cross wavelet spectrum are also shown in Fig. 5e and f, respectively, as generated from the Matlab ${ }^{\circledR}$ wavelet coherence function 'wcoher'. Relative to visual inter-comparison of 
a

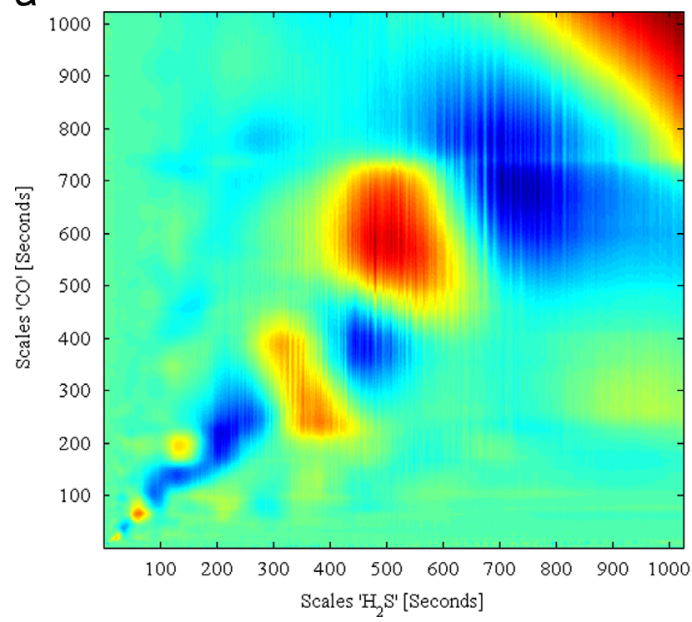

b

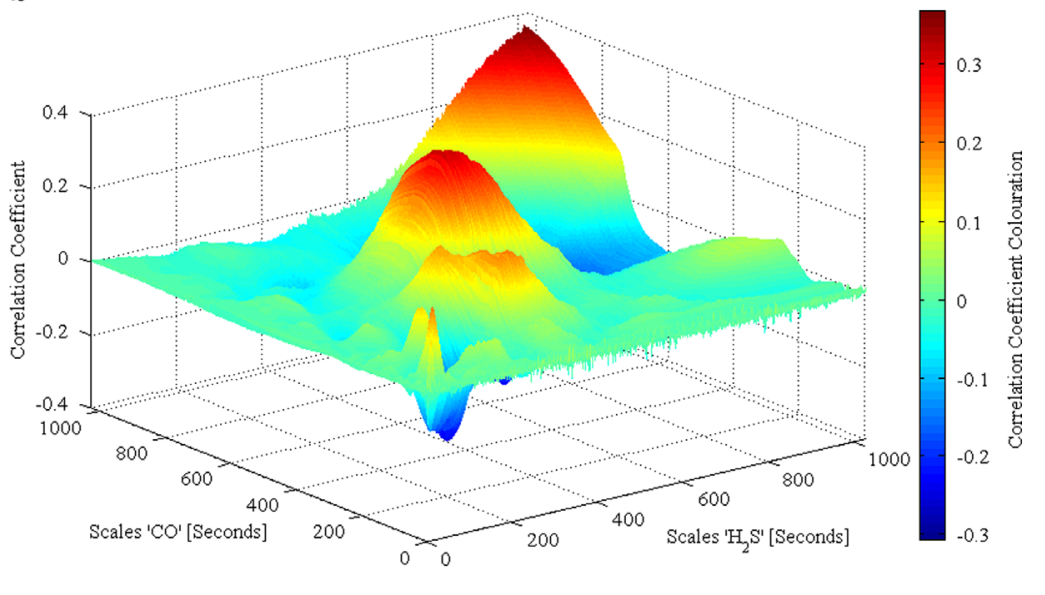

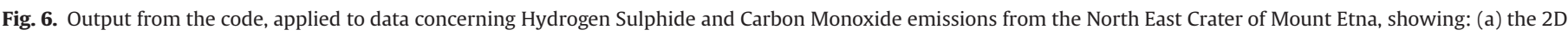
correlation image and (b) the 3D correlation image.

the wavelet plots, or inspection of either of the other two technique outputs, the correlation plot (Fig. 5b) provides scope for far clearer and more intuitive visualisation of the inter-series links, e.g., illustrating the key benefit of the approach over alternates.

Finally, we present the application of our code on volcanic gas signals: Hydrogen Sulphide $\left(\mathrm{H}_{2} \mathrm{~S}\right)$ and Carbon Monoxide (CO) concentration time series, acquired using a 'Multi-GAS' sensor (Shinohara, 2005; Aiuppa et al., 2005) placed in the plume of the North East Crater of Mount Etna (Sicily, Italy). Fig. 6a shows the correlation image generated. The most significant features are positive links between the datasets at $\approx 300-400 \mathrm{~s}, \approx 500-700 \mathrm{~s}$, and at $>900 \mathrm{~s}$. These are similar to the periodicities in sulphur dioxide $\mathrm{SO}_{2}$ emission rates reported by Tamburello et al. (2012) indicating that a variety of volcanic gases fluctuate rapidly in their fluxes, with similar periodicity characteristics. In addition, several weak negative correlation areas also appear at $\approx 100-300 \mathrm{~s}$, $\approx 400-500 \mathrm{~s}$, and $\approx 700-900 \mathrm{~s}$, revealing points worthy of further investigation. This technique is particularly useful on data such as these as links between the series are resolvable, even where sensors might have differing response characteristics (Aiuppa et al., 2005). In Fig. 6b, this correlation image is displayed in 3D.

\section{Summary and conclusions}

Here, we have presented a new use of CWT analysis combined with correlation to determine the similarity between oscillations present in two separate signals. This paper reports on a straightforward to implement Matlab ${ }^{\circledR}$ code, which executes this approach, providing a more readily interpretable visualisation of these links than available from existing alternate techniques, and the coupled capacity to resolve connections between noisy and transient signals. A number of example applications have been presented, via the analysis of synthetic signals and those acquired from various disciplines within the geosciences, which demonstrate the above benefits.

\section{Acknowledgements}

We thank the Editor, Associate Editor and an anonymous reviewer for their consideration and comments upon this paper, which have greatly improved its quality. TDP and AMcG also acknowledge the support of a NERC studentship (x/007002-14), the University of Sheffield and a Google Faculty Research award (r/136958-11-1). AA acknowledges support from the European
Research Council Starting Independent Research Grant (agreement number 1305377). We acknowledge logistical support in the fieldwork by colleagues from the Università degli Studi di Palermo and INGV Catania and Palermo.

\section{Appendix A. Supporting information}

Supplementary data associated with this article can be found in the online version at http://dx.doi.org/10.1016/j.cageo.2014.06.006.

\section{References}

Aiuppa, A., Federico, C., Paonita, A., Giudice, G., Valenza, M., 2005. Chemical mapping of a fumarolic field: La Fossa Crater, Vulcano Island (Aeolian Islands, italy). Geophys. Res. Lett. 13 (L13309), http://dx.doi.org/10.1029/2005GL023207.

Best, D.J., Roberts, D.E., 1975. Algorithm AS 89: the upper tail probabilities of Spearman's rho. Appl. Stat. 24, 377-379.

Cannata, A., Montalto, P., Patanè, D., 2013. Joint analysis of infrasound and seismic signals by cross wavelet transform: detection of Mt. Etna explosive activity. Nat. Hazards Earth Syst. Sci. 13, 1669-1677.

Colestock, M.A., 1993. Wavelets - a new tool for signal processing analysts. In: Proceedings of the 12th AIAA/IEEE Digital Avionics Systems Conference, DASC, pp. 54-59.

Daubechies, I., 1990. The wavelet transform time-frequency localization and signal analysis. IEEE Trans. Inf. Theory 36, 961-1004.

Frohlich, C., Lean, J., 2004. Solar radiative output and its variability: evidence and mechanisms. Astron. Astrophys. Rev. 12 (4), 273-320, http://dx.doi.org/10.1007/ s00159-004-0024-1.

Grinstead, A., Moore, J.C., Jevrejeva, S., 2004. Application of the cross wavelet transform and wavelet coherence to geophysical time series. Nonlinear Process. Geophys. 11, 561-566.

Harris, F.J., 1978. Use of windows for harmonic-analysis with discrete fouriertransform. Proc. IEEE 66 (1), 51-83, http://dx.doi.org/10.1109/PROC.1978.10837.

Hoyt, D.V., Schatten, K.H., 1997. The Role of the Sun in Climate Change. Oxford Univ. Press, Oxford, U.K..

Huang, N.E., Shen, Z., Long, S.R., Lu, M.C., Shih, H.H., Zheng, Q., Yen, N.-C., Tung, C.C., Liu, H.H., 1998. The empirical mode decomposition and the hilbert spectrum for nonlinear and non-stationary time series analysis. Proc.: Math., Phys. Eng. Sci. 454 (1971), 903-995.

Hurrell, J.W., 1995. Decadal trends in the North-Atlantic oscillation - regional temperatures and precipitation. Science 269 (5224), 676-679.

Hurrell, J.W., Kushnir, Y., Ottersen, G., Visbeck, M. (Eds.), 2003. The North Atlantic Oscillation: Climate Significance and Environmental Impact, 134. American Geophysical Union Geophysical Monograph Series, New Jersey, p. 279.

Kendall, M.G., 1970. Rank Correlation Methods, Griffin, London.

Lockwood, M., 2012. Solar influence on global and regional climates. Surv. Geophys. 33 (3-4), 503-534, http://dx.doi.org/10.1007/s10712-012-9181-3.

Morlet, J., Arens, G., Fourgeau, E., Giard, D., 1982. Wave propagation and sampling theory - Part 1: complex signal and scattering in multilayered media. Geophysics 47 (2), 203-221.

Nyquist, H., 2002. Certain topics in telegraph transmission theory (reprinted from Transactions of the AIEE, February, pp. 617-644, 1928). Proc. IEEE 90 (2), 280-305, http://dx.doi.org/10.1109/5.989875. 
Oppenheim, A.V., Schafer, R.W., Buck, J.R., 1999. Discrete-Time Signal Processing, second ed. Prentice Hall, New Jersey.

Pering, T.D., Tamburello, G., McGonigle, A.J.S., Aiuppa, A., Cannata, A., Giudice, G., Patanè, D., 2014. High time resolution fluctuations in volcanic carbon dioxide degassing from Mount Etna. J. Volcanol. Geotherm. Res. 270, 115-121, http://dx. doi.org/10.1016/j.jvolgeores.2013.11.014.

Philander, S.G., 1990. El Niño, La Niña, and the Southern Oscillation, vol. 46. Elsevier International Geophysics Series, Sand Diego, pp. iii-ix (1-289).

Ramsey, P.H., 1989. Critical values for Spearman's rank order correlation. J. Educ. Behav. Stat. 14 (3), 245-253, http://dx.doi.org/10.3102/10769986014003245.

Shinohara, H., 2005. A new technique to estimate volcanic gas composition: plume measurements with a portable multi-sensor system. J. Volcanol. Geotherm. Res. $143,319-333$.
Spearman, C., 1904. The proof and measurement of association between two things. Am. J. Psychol. 15 (1), 72-101.

Tamburello, G., Aiuppa, A., Kantzas, E.P., McGonigle, A.J.S., Ripepe, M., 2012. Passive vs. active degassing modes at an open-vent volcano (Stromboli, Italy). Earth Planet. Sci. Lett. 359-360, 106-116.

Torrence, C., Compo, G.P., 1998. A practical guide to wavelet analysis. Bull. Am. Meteorol. Soc. 79 (1), 61-78.

Welch, P.D., 1967. The use of fast fourier transform for the estimation of power spectra: a method based on time averaging over short, modified periodograms. IEEE Trans. Audio Electroacoust. AU-15 (2), 70-73.

Zar, J.H., 1972. Significance testing of the Spearman rank correlation coefficient. J. Am. Stat. Assoc. 67, 578-580. 